\title{
Relação entre Triatoma infestaans, Aves Domésticas e o Homem num Povoado de Santiago del Estero, Argentina ${ }^{1}$
}

\author{
The Relationship Between Triatoma infestans, Poultry, and Humans in a \\ Rural Settlement in Santiago del Estero, Argentina
}

\author{
Patrícia P. Gajater, María V. Bottazzí, \\ Silvia M. Pietrokovsky², Cristina Wisnivesky-Coll?
}

\begin{abstract}
GAJATE, P. P.; BOTTAZZI, M. V.; PIETROKOVSKY, S. M. \& WISNIVESKY-COLLI, C. The Relationship Between Triatoma infestans, Poultry, and Humans in a Rural Settlement in Santiago del Estero, Argentina. Cad. Saúde Públ., Rio de Janeiro, 10(1): 53-57, Jan/Mar, 1994.
\end{abstract}

An association was determined between Triatona infestans, poultry, and humans in Trinidad, in the Province of Santiago del Estero, Argentina. To collect triatomines, four samples were taken at the area immediately surrounding six houses by the one hour/man capture method (December 1991 - October 1992). Peridomiciliary ecotopes were classified as arthropic (where humans carry out daily activities) or non-anthropic. Triatomine feeding habits were also determined. Of 134 biotopes, $21 \%$ had T. infestans, 22\% had poultry, and $54 \%$ were anthropic. Some $25 \%$ of

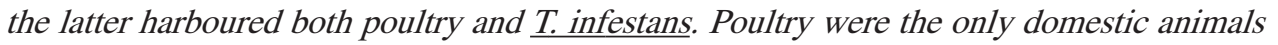
associated with $\underline{T}$. infestans, a finding that was exclusive to anthropic ecotopes. The proportion of feedings on individual fowl (61/146) was highly significant.

Chicken coops are not used in Trinidad, and poultry brood in anthropic structures. Due to the materials used for making their nests and their repeated use, a periodic bug flow can be established from the intra- to the peridomiciliary area and vice versa, through passive transport. The close relationship among $\underline{T}$. infestans, poultry, and humans - in anthropic biotopes where other T. cruzi reservoirs such as dogs are also present - contributes to the maintenance of domestic triatomine colonies and transmission of Chagas' disease to humans.

Key words: Triatoma infestans; Poultry; Anthropic Environment; Chagas' Disease; Santiago del Estero

\section{INTRODUÇÃO}

Na Argentina, onde o vetor mais importante da doença de Chagas é o Triatoma infestans (WHO, 1991), a Província de Santiago del Estero registra o maior índice de endemicidade.

$\mathrm{O} T$. infestans está altamente adaptado a ecótopos artificiais, tanto domésticos como peridomésticos (Ronderos et al., 1980), sendo

\footnotetext{
${ }^{1}$ Trabalho realizado com auxílio da Secretaría de Ciencia y Técnica de la Universidad de Buenos Aires. ${ }^{2}$ Unidad de Ecología de Reservorios y Vectores de Parásitos, Dep. de Biología, Facultad de Ciencias Exactas y Naturales - UBA, Ciudad Universitaria, C. P. 1428, Buenos Aires, Argentina
}

muito freqüente encontrá-lo em galinheiros (Bermúdez et al., 1978; Wisnivesky-Colli et al., 1982; Salvatella, 1986). Muynck et al. (1978) observaram uma relação entre a abundância deste triatomíneo e a presença de galinhas em Santa Cruz de la Sierra, Bolívia.

As aves domésticas representam uma importante fonte de alimentação, atuando como mantenedoras de colônias de triatomíneos (Wisnivesky-Colli et al., 1987). Rossel (1984) sugeriu que as galinhas são facilmente detectadas, dadas sua elevada temperatura corporal e sua alta relação superfície/volume. Numa área rural de Santiago del Estero determinou-se que o perfil alimentar das populações de $T$. infestans domiciliares apresentava variações, depen- 
dendo da época do ano, predominando as ingestas em aves só nos meses quentes. Isto poderia se atribuído ao fato de incubação dos ovos realizar-se no intradomicílio (Wisnivesky-Colli et al., 1987).

No entanto, não há estudos de campo que demonstrem a existência de uma relação entre a presença de $T$. infestans e a forma de criação das aves domésticas.

Este trabalho tem por objetivo determinar a associação entre T. infestans e aves domésticas, bem como a relação destas com o homem, em Santiago del Estero, onde não se verifica a existência de galinheiros.

\section{MATERIAL E MÉTODOS}

O local selecionado para o estudo foi o povoado de Trinidad $\left(27^{\circ} \mathrm{S}, 63^{\circ} \mathrm{O}\right)$, Departamento de Moreno, Província de Santiago del Estero, noroeste da Argentina.

O povoado de Trinidad está formado por 19 casas tipo cafua - de pau-a-pique e adobe -, circundadas por um bosque secundário de "quebracho colorado" (Schynopsis lorentzii) e "quebracho blanco" (Aspidosperma quebracho blanco).

A amostra correspondeu às áreas peridomiciliares de seis casas. As estruturas peridomiciliares mais freqüentes são currais de cabras, porcos, cavalos e vacas (construções abertas feitas de ramada e/ou pau); cozinhas, banheiros e depósitos (estruturas fechadas, geralmente com paredes de adobe); e fornos de tijolo. Não se verifica a existência de estruturas tipo galinheiros, sendo por isso freqüente encontrar as aves domésticas em diferentes locais do peridomicílio.

Para a coleta de triatomíneos foram realizadas quatro amostragens entre dezembro de 1991 e outubro de 1992, através do método de captura hora/homem, com substâncias desalojante tetrametrina a 0,2\% (Wisnivesky-Colli et al., 1987). Devido ao fato dos moradores modificarem periodicamente seus anexos, estes foram considerados como independentes em cada amostragem. Os triatomíneos coletados foram colocados em sacos plásticos com papel absorvente, identificando-se, no rótulo, o domicílio, o ecótopo e a fonte de alimentação nele presen- te. No laboratório identificou-se a espécie com a utilização da chave dicotômica de Lent \& Wygodzinsky (1979) e Brewer et al. (1983).

O perfil alimentar foi determinado pelo teste de difusão dupla em gel de agar, com o conteúdo do mesentério dos barbeiros contra antisoros específicos, correspondentes à fauna local. Estes foram fabricados em coelhos, utilizando-se proteínas sangüíneas de aves, Dipelphidae, Cricetidae, Dasipodidae, Cavidae, Mustelidae, Bovidae, Suidae, Equidae, Canidae, Felidae, répteis e seres humanos (Wisnivesky-Colli et al., 1982).

Com a finalidade de verificar a conexão entre o homem, aves domésticas e T. infestans, os ecótopos foram classificados quanto à sua antropicidade, em antrópicos e não-antrópicos. Definiram-se como antrópicos aqueles anexos onde o homem realizava atividades cotidianas, estando os mesmos localizados próximo ao domicílio, isto é, a menos de 20 metros (cozinhas, banheiros, fornos, etc.), e como nãoatrópicos as estruturas abertas, onde a presença do homem só se verifica ocasionalmente (currais).

Para se determinar a existência de uma associação entre ecótopo com ave e com $T$. infestans, utilizou-se o teste de Mantel-Haenszel, realizando-se a estratificação segundo a antropicidade do ecótopo. Para se determinar a fonte de alimentação mais importante dos triatomíneos, utilizou-se o teste de proporções múltiplas (Fleiss, 1981).

A densidade de barbeiros não foi determinada devido à heterogeneidade da amostragem, pois a complexidade das estruturas não o permitiu. Por outro lado, embora tenha sido estimada a abundância relativa em cada local, seria impossível fazer a comparação da mesma entre os diferentes anexos.

\section{RESULTADOS}

De um total de 134 ecótopos investigados, 28 (21\%) estavam infestados com $T$. infestans e 30 (22\%) tinham aves domésticas. Observou-se uma associação entre $T$. infestans e a presença de aves domésticas $(\mathrm{p}<0,001)$, associação esta que não se repetiu com outros animais domésticos. Do total de ecótopos examinados, 73 
(54\%) eram antrópicos. Destes, 18 (25\%) possuíam aves e $T$. infestans simultaneamente (Tabela 1).

A análise estratificada segundo a antropicidade do ecótopo mostrou que a associação entre presença de aves domésticas e $T$. infestans está fortemente relacionada com os ecótopos antrópicos (Tabela 1).

Através do conteúdo alimentar determinou-se que $91(62,3 \%)$ dentre 146 espécimes de $T$. infestans haviam-se alimentado em aves, sendo esta proporção altamente significativa $(\mathrm{p}<$ 0,001 ), o que não ocorreu com outras fontes de alimentação. Dos 146 barbeiros só 3 haviam se alimentado em seres humanos -2 dos quais também em aves -, todos pertencentes ao mesmo anexo (cozinha).

\section{DISCUSSÃO}

Visto que na Província de Santiago del Estero inexistem estruturas tipo galinheiros, as aves domésticas dormem em árvores, embora, quando estão chocando ou são pequenas, os habitantes do povoado as coloquem em locais protegidos (tanto no peridomicílio como no intradomicílio) freqüentados por eles. Isto faz com que as aves tornem-se muito vulneráveis ao ataque de predadores selvagens. Por outro lado, o homem tem que alimentá-las, diferentemente do que ocorre com os outros animais de criação, que procuram o alimento no mato.
A inexistência de galinheiros poderia ser explicada por fatores sócio-econômicos e culturais. A construção destes anexos é de difícil implementação, pois seus custos são inacessíveis aos moradores. Em Trinidad, o uso que se faz das aves domésticas está relacionado com o tipo de economia local (de subsistência). Em geral, os animais são criados por cada família em números reduzidos, sendo os mesmos utilizados para o consumo interno só quando já estão velhos para pôr ovos ou para se reproduzir. Também as características climáticas da área influenciam este uso: a água é sempre um recurso escasso, especialmente na estação seca, quando os habitantes restringem de tal forma o seu uso que todos os animais domésticos têm de procurá-la por si mesmos, em locais afastados da casa.

A estreita associação observada entre galinhas e T. infestans nos ecótopos peridomésticos mais visitados pelo homem sugeriria um certo risco para este último. Segundo observações feitas em campo, os habitantes do povoado preparavam os ninhos para as aves utilizando caixas de papelão, roupa velha, trapos, etc. Estes elementos, onde foi coletada a maioria dos barbeiros, encontravam-se no anexo peridomiciliar ou eram trazidos das casas. Portanto, alguns triatomíneos poderiam ser provenientes do intradomicílio, e as galinhas, como fonte de alimentação, permitiriam a manutenção das colônias no peridomicílio. Terminada a época de incubação, as aves abandonavam o ninho e estes elementos

TABELA 1. Freqüência de Ecótopos Peridomésticos segundo a Antropicidade, Presença de Aves Domésticas e de Triatoma infestans no Povoado de Trinidad, Departamento de Moreno, Província de Santiago del Estero, Argentina (1991-1992)

\begin{tabular}{|c|c|c|c|c|c|}
\hline \multicolumn{6}{|c|}{ Tipo de Ecótopo* } \\
\hline & \multicolumn{2}{|c|}{ Antrópico } & \multicolumn{2}{|c|}{ Não-Antrópico } & \\
\hline & \multicolumn{2}{|c|}{ T. infestans } & \multicolumn{2}{|c|}{ T. infestans } & Total \\
\hline Ave & Sim & Não & Sim & Não & \\
\hline Presente & 18 & 11 & 0 & 1 & 30 \\
\hline Ausente & 2 & 42 & 8 & 52 & 104 \\
\hline Nível de Significância & \multicolumn{2}{|c|}{$\mathrm{p}<0,001$} & \multicolumn{2}{|c|}{ NS } & \\
\hline \multirow[t]{2}{*}{ Total } & 20 & 53 & 8 & 53 & 134 \\
\hline & \multicolumn{2}{|c|}{73} & \multicolumn{2}{|c|}{61} & \\
\hline
\end{tabular}

* Tipo de ecótopo: classificação segundo a antropicidade, antrópico: ecótopo onde o homem realiza atividades cotidianas, localizado próximo ao domicílio. 
poderiam ser então levados para outros locais da casa para serem reutilizados. Assim, se estabelecer-se-ia um fluxo periódico de barbeiros do intradomicílio para o peridomicílio, e vice-versa, através do transporte passivo. Tal situação tornar-se-ia particularmente perigosa após uma borrifação com inseticida, pois o peridomicílio atuaria como foco residual de triatomíneos, permitindo a recolonização das casas (Forattini et al.,1983).

Por outro lado, nos ecótopos do tipo antrópico também existem outras fontes importantes do $T$. cruzi (por exemplo cães), favorecendo a transmissão do parasito ao homem (WisniveskyColli et al., 1985).

A construção de galinheiros, de um ponto de vista epidemiológico, implicaria a aglomeração de aves domésticas num único anexo, originando densidades triatomínicas muito elevadas (Bermúdez et al., 1978) e persistentes, o que resultaria em um difícil controle. Por este motivo, acredita-se que a construção de galinheiros só contribuiria para o controle do vetor caso fossem realizadas borrifações periódicas com inseticida, o que não tem ocorrido até o momento.

\section{AGRADECIMENTOS}

Agradecemos às comunidades de Amamá e Trinidad pela sua colaboração, em especial ao Sr. Juan Moyano. Agradecemos também à Lic. Tatiana Sosa pela ajuda na correção do trabalho.

\section{RESUMO}

GAJATE, P. P.; BOTTAZZI, M. V.; PIETROKOVSKY, S. M. \& WISNIVESKYCOLLI, C. Relação entre Triatoma infestans, Aves Domésticas e o Homem num Povoado de Santiago del Estero, Argentina. Cad.Saúde Públ., Rio de Janeiro, 10(1): 53-57, jan/mar, 1994.

Determinou-se a associação entre Triatoma infestans, aves domésticas e o homem no povoado de Trinidad, Santiago del Estero, Argentina.

Para coletar triatomíneos foram realizadas quatro amostragens no peridomicílio de seis casas, utilizando-se o método de captura hora/homem (dezembro de 1991 a outubro de 1992). Os anexos foram classificados em antrópicos (onde o homem realiza atividades cotidianas) e não-antrópicos. Além disso, determinou-se o perfil alimentar dos barbeiros. De um total de 134 ecótopos investigados, 21\% tinham T.infestans; $22 \%$ possuíam aves domésticas; e 54\% eram antrópicos. Em 25\% destes ecótopos foram encontradas aves e $T$. infestans simultaneamente. As aves foram os únicos animais domésticos associados com o T. infestans, sendo que esta relação só ocorreu nos ecótopos antrópicos. A proporção de ingestas em aves (61/146) foi altamente significativa.

Em Trinidad não há galinheiros, utilizando-se, assim, os anexos entrópicos do peridomicílio como local para se construir os ninhos para as aves. tanto pelos elementos usados na preparação dos ninhos como pelo uso posterior dos mesmos, poder-se-ia estabelecer um fluxo periódico de barbeiros do intradomicílio para o peridomicílio, e vice-versa, através do transporte passivo.

Esta relação estreita entre Triatoma infestans, aves domésticas e o homem, encontrada nos ecótopos antrópicos, onde também existem outros reservatórios de T. cruzi, como os cães, favorece a manutenção de colônias de barbeiros domiciliares e a transmissão da doença de Chagas ao homem.

Palavras-Chave: Triatoma infestans; Aves Domésticas; Ambiente Antrópico; Doença de Chagas; Santiago del Estero 


\section{REFERÊNCIAS BIBLIOGRÁFICAS}

BERMÚDEZ, H. GARRON, A. \& MUYNCK, A., 1978. Infestación del peridomicilio por Triatoma infestans en Gutierrez (Província Cordillera, Departamento Santa Cruz). Disección de un gallinero. Boletin Informativo del Centro Nacional de Enfermedades Tropicales, 4: 48-51.

BREWER, M.; GORLA, D. K. \& GARAY, M. E., 1983. Caracterización de los estadios ninfales del género Triatoma Laporte, 1983. II Análisis biométrico descriptivo de Triatoma infestans Klug, 1834; Triatoma platensis Neiva, 1913; Triatoma delpontei Romaña y Avalos, 1947 y Triatoma sordida Stal, 1859 (Hemiptera, Reduviidae). Revista de la Sociedad de Entomología Argentina, 42: 81-88.

FLEISS, J. L., 1981. Assessing significance in a fourfold table. In: Statistical Methods for Rates and Proportions (R. A. Bradley; J. S. Hunter; D. G. Kendall \& G. S. Watson, eds.), $2^{a}$ ed., pp. 19-32, New York John Wiley \& Sons. (Wiley Series in Probability and Mathematical Statistic)

FORATTINI, O. P.; FERREIRA, O. A.; RABELLO, E. X.; SOARES-BARATA, J. M. \& SANTOS, J. L. F., 1983. Aspectos ecológicos da tripanossomíase americana. XVIII Desenvolvimento e ciclos anuais de colônias de Triatoma infestans, T. sordida e Rhodnius neglectus em ecótopos artificiais, no ambiente peri e extradomiciliar. Revista de Saúde Pública, 17: 243-262.

LENT, H. \& WYGODZINSKY, P., 1979. Revision of the triatominae (Hemiptera, Reduviidae) and their significance as vectors of Chagas' disease. Bulletin of the American Museum of Natural History, 163: 123-520.

MUYNCK, A.; RIBERA, B.; ZUNA, H.; VILARROEL, G.; MELGAR, B.; LAGRAVA, M.; LAGRAVA, M. S.; NAVIA, J.; VARGAS, J. \& SERRANO, R., 1978. Encuesta epidemiológica en Vallegrande. In: Enfermedad de Chagas (A. R. Dávalos, org.), pp. 115-150, La Paz-Cochabamba: Los Amigos del Libro.
RONDEROS, R. A. L SCHNACK, J. A. \& MAURI, R. A., 1980. Resultados preliminares respecto de la ecología de Triatoma infestans (Klug) especies congenéricas con referencia especial a poblaciones peridomiciliarias. Medicina, 40: 187196.

ROSSEL, O. R., 1984. A comparison of the feeding performances of Rhodnius prolixus Stal and Rhodnius robustus Larrousse (Hemiptera: Triatominae). Thesis of Doctor of Philosophy, London: Departament of Pure and Applied Biology, Imperial College of Science and Technology, University of London.

SALVATELLA, R., 1986. Triatominos del Uruguay. Revista de Medicina de Uruguay, 2: 106-113.

WHO (World Health Organization), 1991. Control of Chagas' Disease. Report of a WHO Expert Commitee. Geneva: WHO.

WISNIVESKY-COLLI, C.; GÜRTLER, R. E.; SOLARZ, N. D.; SALOMON, D. D. \& RUIZ, A. M., 1982. Feeding patterns of Triatoma infestans (Hemiptera, Reduviidae) in relation to transmission of American trypanosomiasis in Argentina. Fournal of Medical Entomology, 19: 645654.

WISNIVESKY-COLLI, C.; GÜRTLER, R. E., SOLARZ, N. D.; LAURICELLA, M. A. \& SEGURA, E. L., 1985. Epidemiological role of humans, dogs and cats in the transmission of Trypanosoma cruzi in a central area of Argentina. Revista do Instituto de Medicina Tropical, 27: 346-352.

WISNIVESKY-COLLI, C.; RUIZ, A. M.; LEDESMA, O.; GÜRTLER, R. E.; LAURICELLA, $M$. A.; SALOMON, D. D.; SOLARZ, N. D. \& SEGURA, E.L., 1987. Ecologia doméstica de la tripanosomiasis americana: Perfil alimentario del Triatoma infestans en un área rural de Santiago del Estero. Revista da Sociedade Brasileira de Medicina Tropical, 20: 31-39. 